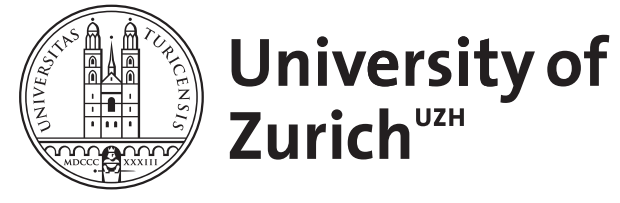

\title{
Cardiac hybrid imaging
}

\author{
Gaemperli, Oliver ; Kaufmann, Philipp A ; Alkadhi, Hatem
}

\begin{abstract}
Hybrid cardiac single photon emission computed tomography (SPECT)/CT imaging allows combined assessment of anatomical and functional aspects of cardiac disease. In coronary artery disease (CAD), hybrid SPECT/CT imaging allows detection of coronary artery stenosis and myocardial perfusion abnormalities. The clinical value of hybrid imaging has been documented in several subsets of patients. In selected groups of patients, hybrid imaging improves the diagnostic accuracy to detect CAD compared to the single imaging techniques. Additionally, this approach facilitates functional interrogation of coronary stenoses and guidance with regard to revascularization procedures. Moreover, the anatomical information obtained from CT coronary angiography or coronary artery calcium scores (CACS) adds prognostic information over perfusion data from SPECT. The use of cardiac hybrid imaging has been favoured by the dissemination of dedicated hybrid systems and the release of dedicated image fusion software, which allow simple patient throughput for hybrid SPECT/CT studies. Further technological improvements such as more efficient detector technology to allow for low-radiation protocols, ultra-fast image acquisition and improved low-noise image reconstruction algorithms will be instrumental to further promote hybrid SPECT/CT in research and clinical practice.
\end{abstract}

DOI: https://doi.org/10.1007/s00259-013-2566-9

Posted at the Zurich Open Repository and Archive, University of Zurich

ZORA URL: https://doi.org/10.5167/uzh-94293

Journal Article

Published Version

Originally published at:

Gaemperli, Oliver; Kaufmann, Philipp A; Alkadhi, Hatem (2014). Cardiac hybrid imaging. European Journal of Nuclear Medicine and Molecular Imaging, 41(S1):91-103.

DOI: https://doi.org/10.1007/s00259-013-2566-9 


\title{
Cardiac hybrid imaging
}

\author{
Oliver Gaemperli • Philipp A. Kaufmann • \\ Hatem Alkadhi
}

Received: 24 August 2013 / Accepted: 27 August 2013 / Published online: 22 March 2014

(C) Springer-Verlag Berlin Heidelberg 2014

\begin{abstract}
Hybrid cardiac single photon emission computed tomography (SPECT)/CT imaging allows combined assessment of anatomical and functional aspects of cardiac disease. In coronary artery disease (CAD), hybrid SPECT/CT imaging allows detection of coronary artery stenosis and myocardial perfusion abnormalities. The clinical value of hybrid imaging has been documented in several subsets of patients. In selected groups of patients, hybrid imaging improves the diagnostic accuracy to detect CAD compared to the single imaging techniques. Additionally, this approach facilitates functional interrogation of coronary stenoses and guidance with regard to revascularization procedures. Moreover, the anatomical information obtained from CT coronary angiography or coronary artery calcium scores (CACS) adds prognostic information over perfusion data from SPECT. The use of cardiac hybrid imaging has been favoured by the dissemination of dedicated hybrid systems and the release of dedicated image fusion software, which allow simple patient throughput for hybrid SPECT/CT studies. Further technological improvements such as more efficient detector technology to allow for low-radiation protocols, ultra-fast image acquisition and improved low-noise image reconstruction algorithms will be instrumental to further promote hybrid SPECT/CT in research and clinical practice.
\end{abstract}

Keywords Myocardial perfusion SPECT · Coronary CT angiography $\cdot$ Hybrid imaging

O. Gaemperli • P. A. Kaufmann

Cardiac Imaging, University Hospital Zurich, Zurich, Switzerland

H. Alkadhi

Institute of Diagnostic and Interventional Radiology,

University Hospital Zurich, Zurich, Switzerland

O. Gaemperli $(\bowtie)$

Nuclear Cardiology, Cardiovascular Center, University Hospital Zurich, NUK C 40, Raemistrasse 100, 8091 Zurich, Switzerland e-mail: oliver.gaemperli@usz.ch

\section{Introduction}

Non-invasive cardiac imaging has witnessed tremendous advances in the last few decades. This holds true particularly for techniques to detect coronary artery disease (CAD) which are currently used for the diagnosis, prognostication and risk stratification of patients with known or suspected $\mathrm{CAD}$. As a result, the number of non-invasive cardiac imaging tests has steadily increased over the last few years [1]. This has driven the dissemination of state-of-the art high-end devices for computed tomography (CT) and nuclear cardiology worldwide.

Besides morphological CAD, functional consequences of coronary stenoses on myocardial perfusion and metabolism are relevant. Additionally, other entities such as microvascular or endothelial dysfunction have been recognized to contribute significantly to disease pathology [2, 3]. Hence, clinical cardiac imaging is increasingly challenged to address in a comprehensive manner the anatomical and functional aspects of $\mathrm{CAD}$ in order to provide guidance for appropriate medical or interventional therapies.

Hybrid imaging has achieved increasing clinical acceptance because it provides anatomical information on the presence of coronary artery lesions combined with functional information on its pathophysiological consequences with regard to myocardial perfusion or metabolism. The present review provides an overview on the most important clinical aspects of cardiac single photon emission computed tomography (SPECT)/CT hybrid imaging touching upon the clinical need and the technical requirements. We provide an overview of relevant literature highlighting the clinical value and addressing potential indications and pitfalls of cardiac hybrid imaging. Finally, as research is ongoing and the technique is continuously expanding to other indications we will provide a glimpse into potential future clinical avenues of hybrid imaging. 


\section{Clinical background}

There is poor agreement between the degree of stenosis of a coronary lesion and the presence of myocardial ischaemia. A number of studies using intraoperative Doppler ultrasound, positron emission tomography (PET) and fractional flow reserve (FFR) have consistently demonstrated that the angiographic severity of coronary lesions is a poor predictor of its haemodynamic relevance [4-7]. Similarly, comparative studies of CT coronary angiography (CTCA) and myocardial perfusion imaging (MPI) showed that only $30-50 \%$ of stenoses with more than $50 \%$ luminal narrowing are associated with reversible perfusion defects on MPI, and conversely many patients with completely normal myocardial perfusion may harbour subclinical CAD [8-11].

Large randomized clinical trials have failed to demonstrate superiority of revascularization over modern medical treatment in terms of long-term cardiovascular events if patients are not stratified by prior ischaemia testing $[12,13]$. Reducing the ischaemic burden of the left ventricular (LV) myocardium seems to improve the outcome of stable CAD patients [14, 15]. The pivotal role of ischaemia-targeted revascularization has further been highlighted in the randomized FAME I trial [16]. Finally, the FAME II trial has proven - for the first time in a prospective randomized study - superiority of revascularization over optimal medical treatment for patients with flowlimiting coronary stenoses (FFR $<0.80)$ [17]. Similar large prospective randomized trials with non-invasive imaging instead of FFR are currently enrolling patients [18]. Consequently, tests for myocardial ischaemia are incorporated into current revascularization guidelines, beyond the assessment of coronary morphology alone [19].

Thus, functional interrogation of coronary lesions is feasible with FFR during invasive coronary angiography and has gained wide acceptance based on the solid evidence published in favour of the technique. However, a non-invasive anatomical and functional assessment of CAD should be preferred as first-line test in the majority of patients with suspected CAD in order to reduce procedure-related morbidity and costs [20]. $\mathrm{CT}_{\mathrm{FFR}}$ was recently developed as a method to determine haemodynamic relevance of coronary lesions from standard $\mathrm{CT}$ angiograms using computational fluid dynamics. This technique is still experimental and a recent study has shown limitations of this technique in routine clinical practice [21].

\section{CT coronary angiography}

Accuracy for detection of coronary artery stenosis

The diagnostic performance of CTCA as compared to invasive coronary angiography has been documented in various studies [22] and recently also in meta-analyses [23]. The latter revealed a pooled area under the receiver-operating characteristic curve of 0.99 for diameter stenoses $>50 \%$. Pooled sensitivity and specificity were 99 and $89 \%$, respectively $[24,25]$. The available data consistently document excellent diagnostic performance of CTCA for identifying and ruling out coronary artery stenoses $>50 \%$ when compared to invasive coronary angiography.

Early studies on CTCA reported effective radiation dose levels up to $20 \mathrm{mSv}$ when using spiral acquisitions with retrospective ECG gating. However, current radiation dose levels are far lower, owing to the development and application of sequential and high-pitch scans [26] as well as to the use of lower tube voltage settings and iterative reconstructions [27]. Currently, effective radiation dose levels of CTCA are in the range of $1-5 \mathrm{mSv}$. Given the more widespread application of CTCA with increasing numbers of examinations worldwide, this dose reduction is important for patient safety, particularly if hybrid imaging protocols are considered.

While robustness and stability of CTCA continues to improve with each CT technology development, giving rise to improved temporal and spatial resolution in in vitro studies [28] and a reduction of image noise when using iterative reconstructions [29], CTCA still does not perform well at high and irregular heart rates, in morbidly obese patients, in patients with stents and in cases of severe coronary calcifications. Each of these features may result in a non-diagnostic CTCA study, precluding its application in these patients.

\section{Plaque detection and characterization}

Being a cross-sectional imaging modality, CTCA depicts the coronary wall including plaques in addition to stenoses. Most studies so far have focused on a simple categorization scheme of coronary plaques as non-calcified, partially calcified and calcified. By doing so, authors found a good agreement between CTCA and intravascular ultrasound representing the clinical reference standard modality for plaque characterization. Still, this current way of plaque classification based on CTCA falls short of a more advanced description of plaque characteristics, such as the identification of thin-cap fibroatheromas which are believed to represent late stage and potentially vulnerable coronary plaques. Recently, Maurovich-Horvat and colleagues described in their in vitro studies a "napkin-ring" sign plaque in coronary arteries, representing a ring of hyperattenuation surrounding a low attenuating centre [30]. This plaque type was highly predictive of thin-cap fibroatheroma as determined by histopathology [30-32]. Interestingly, a recent in vivo study demonstrated that the napkin-ring sign plaque depicted with CTCA was strongly associated with future acute coronary syndrome events, being independent of other high-risk coronary CTCA features [33]. While these recent studies are highly promising, the current clinical role of coronary plaque characterization with CTCA has still not been defined. Further 
improvements in spatial and contrast resolution are required for a better anatomical depiction and differentiation of the various plaque types.

\section{Myocardial perfusion SPECT}

Myocardial perfusion can be assessed using radionuclide imaging studies such as SPECT or (in more specialized centres) PET. With SPECT a vast amount of data is available demonstrating its excellent diagnostic accuracy for detecting angiographically significant CAD with a sensitivity and specificity of $87-89 \%$ and $73-75 \%$, respectively, depending on the choice of radionuclide and stress modality [34]. Gated SPECT offers information of LV volumes and ejection fraction. A large body of evidence documents the prognostic value of SPECT with a consistent $<1 \%$ cardiac event rate for patients with completely normal perfusion on SPECT [35]. Current software packages allow semiquantitative assessment of perfusion defect extent in relation to the total LV myocardium. Based on these quantitative measures of ischaemic burden, SPECT has demonstrated its value for guiding revascularization procedures in CAD patients $[15,19,36]$. In patients with ischaemia extending over $\geq 10 \%$ of the left ventricle, a revascularization procedure improves the outcome, while in patients with $<10 \%$ medical therapy is equivalent or better. Finally, SPECT provides three-dimensional (3-D) data sets as CTCA (in DICOM). These data sets can be easily superimposed onto CT images (after correcting for the lower matrix size and bigger voxel size) using dedicated fusion software.

Again, radiation exposure is a concern, particularly with SPECT and SPECT/CT. However, low-dose SPECT is feasible with novel solid-state detector cameras and improved iterative image reconstruction algorithms with effective radiation doses in the range of 4-7 $\mathrm{mSv}[37,38]$. Further reduction to $<2 \mathrm{mSv}$ is achievable if stress protocols are performed first and rest images are not required. Other functional modalities [stress echocardiography, stress cardiac magnetic resonance imaging (CMR)] are potentially attractive candidates for hybrid imaging. Both modalities avoid ionizing radiation; however, the two-dimensional image format of echocardiograms is unsuitable for fusion with CTCA. Stress CMR/ CTCA fusion may be possible using fast perfusion sequences and is currently under clinical investigation [39-41].

\section{Hybrid systems}

The first hybrid devices were PET/CT tomographs which gained wide acceptance in oncology for the staging of neoplastic disease. Along this path, similar hybrid devices combining SPECT cameras with high-end CT devices and recently also first experimental hybrid PET/MR have been released. Figure 1 gives an overview of commercially available hybrid
SPECT/CT devices from different manufacturers. Some of these devices offer attractive features for hybrid imaging. The Discovery NM/CT 570c consists of a small footprintdedicated cardiac SPECT camera equipped with solid-state semiconductor detector technology and multi-pinhole collimators. This novel assembly combined with the unique geometry results in an exceptionally high photon sensitivity [37]. It allows shortened acquisition times to 5 min or less [42] and thereby improves patient comfort and throughput for hybrid imaging. The Siemens Symbia TruePoint System is equipped with intelligent collimators that track and centre the heart to improve count sensitivity. The AnyScan SPECT/CT system from Mediso Medical Imaging Systems (Budapest, Hungary) is equipped with a 16-slice CT and can be upgraded to a SPECT/PET/CT hybrid system. The majority of these hybrid devices are available with high-end CT devices; however, it should be emphasized that for coronary $\mathrm{CT}$ angiography the recommended minimum number of slices is 64 . CT machines having less slices have a temporal and spatial resolution that is not high enough to provide a diagnostic image quality of all coronary segments in a sufficient number of patients.

The majority of novel SPECT/CT systems offer improved image reconstruction techniques [37]: These novel algorithms are largely based on modelling the specific geometry of the detectors and the collimator to improve count sensitivity. Furthermore, most systems offer compensation for image resolution loss by accelerated iterative reconstruction. Several manufacturers have taken this approach [e.g. wide beam reconstruction (WBR) by UltraSpect Ltd., Haifa, Israel; Astonish by Philips, Best, The Netherlands; IQ SPECT by Siemens, Erlangen, Germany; and Evolution by GE Healthcare, Milwaukee, WI, USA]. With these improved reconstruction techniques, further reductions in image acquisition times or radiation may be achieved [43].

\section{Post-processing and image analysis software}

In the setting of cardiac hybrid imaging the use of hybrid systems is not necessarily required and less important than for oncology whole-body PET/CT. Nonetheless, in cardiac SPECT/CT imaging, manual superposition and individual correction of data set misalignment is crucial to ensure adequate quality for several reasons: cardiac and respiratory motion, intrinsic disagreement in the size and shape of the left ventricle between non-gated SPECT images (unfortunately gated SPECT images are too noisy for hybrid imaging) and diastole-gated CTCA and 3-D instead of cross-sectional display of fused data sets. Therefore, cardiac hybrid imaging can easily be performed by acquiring data sets on stand-alone systems with subsequent software-based fusion, and in fact this set-up may allow exploitation of the full capacity of the 
Fig. 1 Commercially available hybrid SPECT/CT systems by different manufacturers. a Discovery NM/CT 570 c by GE Healthcare. b Symbia TruePoint SPECT/CT by Siemens Healthcare, Erlangen, Germany. c BrightView XCT system by Philips Healthcare, Best, The Netherlands. (d) AnyScan SPECT/CT system from Mediso Medical Imaging Systems, Budapest, Hungary
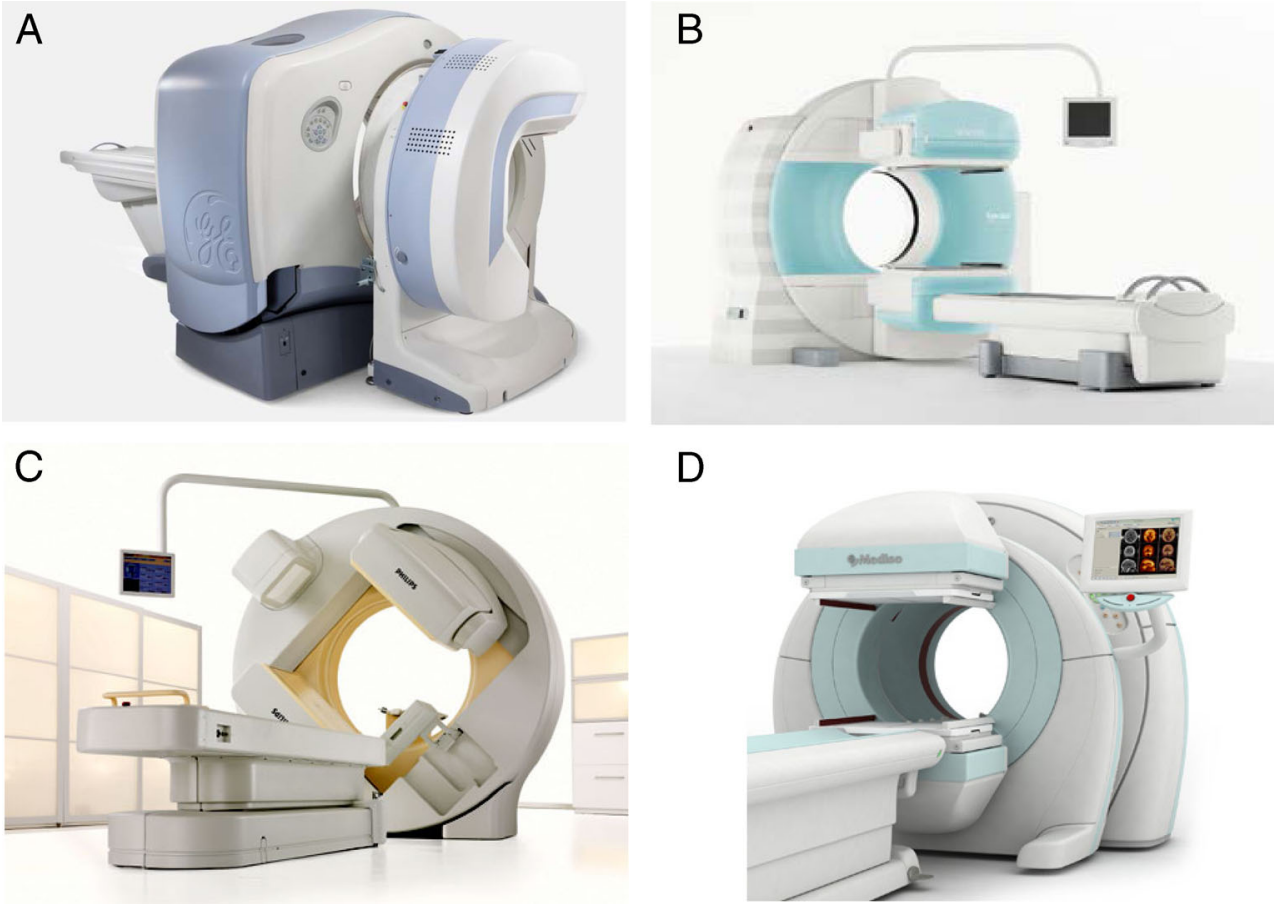

D

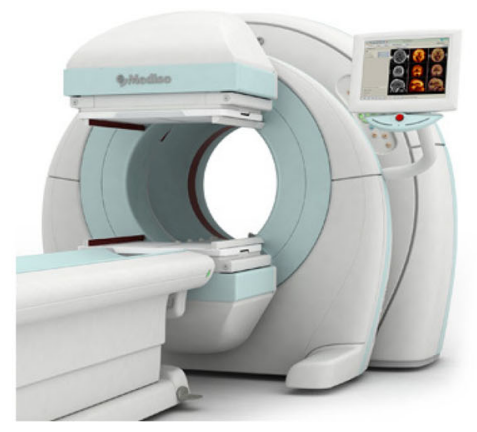

respective systems for all kinds of medical purposes rather than having a highly dedicated hybrid device.

Dedicated software packages are available from different manufacturers/research institutions that allow fusion of CTCA and radionuclide images (Fig. 2) [44]. A crucial element is a user interface that permits correction of misalignment between data sets. The remaining fusion steps include tracking of the coronary arteries (by the same methods as standard CTCA software) and modelling of the perfusion data into a 3-D volume rendering of the left ventricle. Current software packages allow fusion of images from different modalities and even different manufacturers. In experienced hands and with image data sets of reasonable quality, the image fusion process takes no longer than 5 min per patient. Additional reconstruction tools that eliminate the influence of cardiac LV motion in the display and freeze myocardial perfusion information in a diastolic frame ("motion-frozen" technique) may further facilitate image fusion [45].

\section{Clinical value of hybrid imaging}

Table 1 shows a summary of available diagnostic studies with cardiac hybrid imaging. The feasibility and clinical robustness of non-invasive hybrid imaging was first documented by Namdar and co-workers in a clinical study involving fusion of ${ }^{13} \mathrm{~N}_{-N H_{3}}$ PET with 4-slice CTCA in 25 patients with CAD [46]. These encouraging results were replicated with SPECT/ CT in a study by Rispler and co-workers who documented an increase in specificity (from 63 to $95 \%$ ) and positive predictive value (PPV, from 31 to $77 \%$ ) with SPECT/CTCA compared to CTCA alone [47]. Sato and co-workers showed that adding SPECT information in non-evaluable arteries on CTCA improved particularly specificity and PPV significantly (from 80 to $92 \%$ and from 69 to $85 \%$, respectively) [48]. Notably, the majority of non-evaluable severely calcified vessels in the left anterior descending artery were positive on stress nuclear MPI, whereas the majority of non-evaluable vessels with motion artefacts in the right coronary artery were negative. One of the largest, recently published studies included 107 patients undergoing hybrid ${ }^{15} \mathrm{O}-\mathrm{H}_{2} \mathrm{O}$ PET/64-slice CTCA [49]. In this study, haemodynamic significance of coronary stenoses was confirmed in 18 of 40 patients with FFR providing a more comprehensive gold standard. Consequently, the use of PET/CTCA increased the PPV significantly from 76 to $96 \%$ compared to CTCA alone.

However, a number of limitations apply to the aforementioned studies including the limited number of patients, the variety of hybrid systems used and the lack of a uniform gold standard. Three studies have specifically addressed the incremental value of hybrid cardiac imaging over the sideby-side analysis of CTCA and myocardial perfusion images (Table 2) in patients with multivessel disease (MVD), intermediate severity coronary stenoses and/or side branch disease $[45,50,51]$. Hence, hybrid cardiac imaging may facilitate the identification of haemodynamically significant coronary artery stenoses and thereby guide clinicians on the appropriate method of revascularization. Moreover, in intermediate stenoses, a hybrid study may accurately confirm or rule out their haemodynamic significance and thereby reduce the number of 

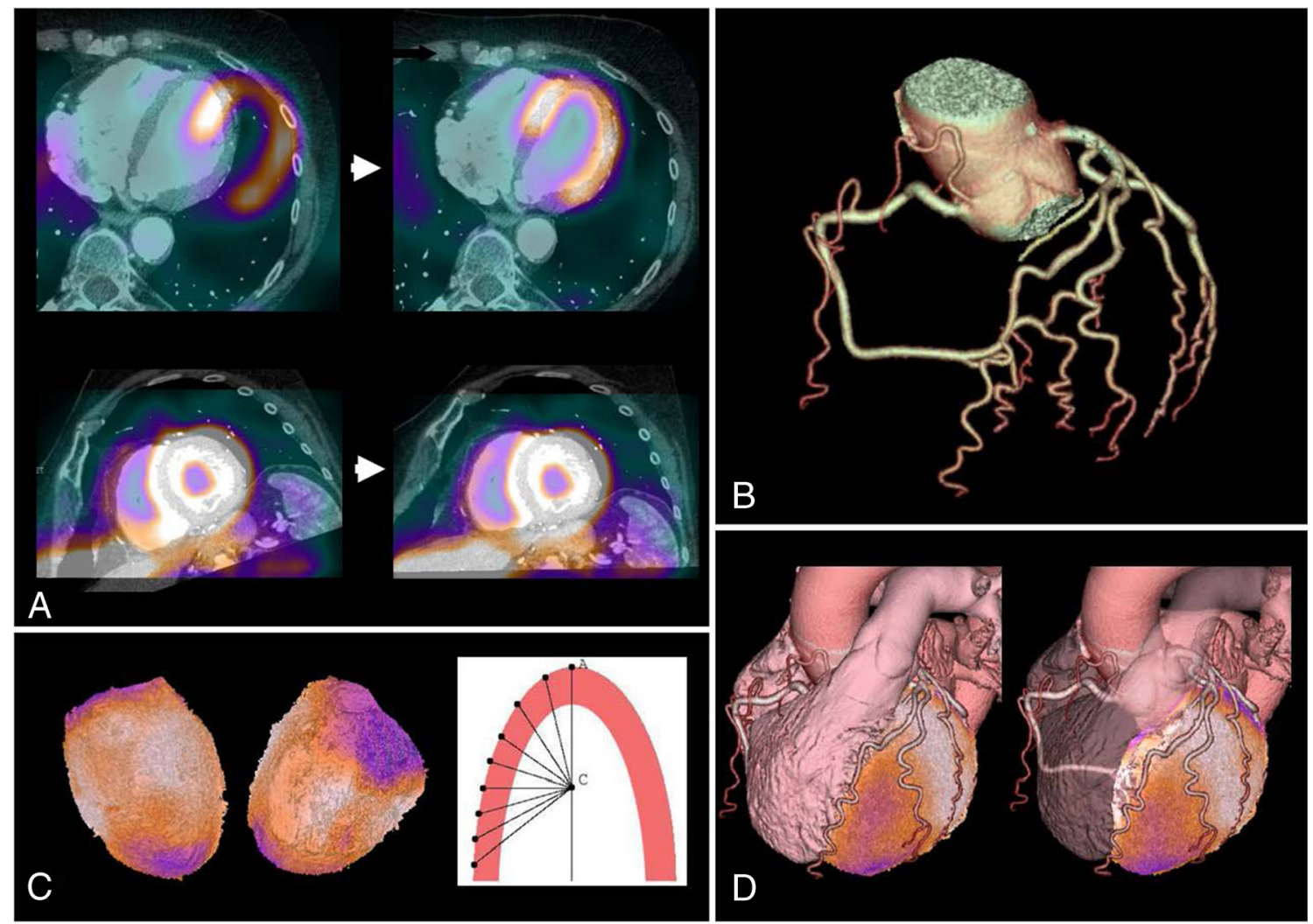

Fig. 2 The main steps of SPECT/CT image fusion (CardIQ Fusion, Advantage Workstation, GE Healthcare). a Image coregistration. This first step is crucial and allows the user to align the images on axial, sagittal and coronal image planes in order to obtain optimal matching of structural and functional information. b Definition of LV epicardium. This protocol displays a view containing the segmented CT LV epicardium using the conventional volume rendering technique, allowing addition or removal of structures from the LV epicardium if needed. In each point of the surface of the volume rendered image the colour is computed as being the

potentially unnecessary stent implantations, a key cost driver in cardiovascular medicine.

A recent study including more than 500 patients has also documented the prognostic value of hybrid imaging [52]. In maximum perfusion intensity on a ray going from the particular point to the centre of the heart on CT. c Coronary tree segmentation. This protocol allows the user to grow the coronary arteries from the ascending aorta using automatic vessel tracking or manual segmentation. d 3-D volume rendered fusion images. As the final step, the hybrid display protocol displays a volume rendering containing the LV epicardial volume, the volume rendered coronary tree, and the left and right heart chambers acquired by an automatic segmentation algorithm. (Reprinted with permission from Springer [44])

this study, CTCA stenoses provided independent prognostic information over SPECT alone. Moreover, the presence of non-calcified plaque emerged as an independent predictor along with CTCA stenoses and abnormal SPECT. Further

Table 1 Added diagnostic accuracy of cardiac hybrid imaging (SPECT/CTCA and PET/CTCA) (vessel-based analysis)

\begin{tabular}{|c|c|c|c|c|c|c|c|}
\hline Author & Hybrid system & $n$ & Gold standard (definition of significant CAD) & Sens & Spec & PPV & NPV \\
\hline Namdar et al. (2005) [46] & ${ }^{13} \mathrm{~N}-\mathrm{NH}_{3} \mathrm{PET} / 4$-slice CTCA & 25 & $\begin{array}{l}\text { Flow-limiting coronary stenoses requiring } \\
\text { revascularization (ICA+PET) }\end{array}$ & 90 & 98 & 82 & 99 \\
\hline Rispler et al. (2007) [47] & SPECT/16-slice CTCA & 56 & $\begin{array}{l}\text { Flow-limiting coronary stenoses } \\
(>50 \% \text { stenosis on ICA }+ \text { SPECT pos. })\end{array}$ & 96 & 95 & 77 & 99 \\
\hline Groves et al. (2009) [82] & ${ }^{82} \mathrm{Rb}$ PET/64-slice CTCA & 33 & $>50 \%$ stenosis on ICA & 88 & 100 & 97 & 99 \\
\hline Sato et al. (2010) [48] & SPECT/64-slice CTCA ${ }^{\mathrm{a}}$ & 130 & $>50 \%$ stenosis on ICA & 94 & 92 & 85 & 97 \\
\hline Kajander et al. (2010) [49] & ${ }^{15} \mathrm{O}-\mathrm{H}_{2} \mathrm{O}$ PET/64-slice CTCA & 107 & $\begin{array}{l}\text { Flow-limiting coronary stenosis } \\
\text { ( }>50 \% \text { stenosis of ICA }+ \text { FFR })\end{array}$ & 93 & 99 & 96 & 99 \\
\hline
\end{tabular}

$n$ number of patients in each study, SPECT single photon emission computed tomography, CTCA CT coronary angiography, PET positron emission tomography, $C A D$ coronary artery disease, Sens sensitivity, Spec specificity, $P P V$ positive predictive value, $N P V$ negative predictive value, $I C A$ invasive coronary angiography, FFR fractional flow reserve

${ }^{a}$ Hybrid SPECT/CTCA only applied for non-evaluable arteries on CTCA (14\%) 
Table 2 Synergistic clinical value of fused hybrid imaging compared to the side-by-side analysis

\begin{tabular}{|c|c|c|c|}
\hline Author & Hybrid system & Patient population & Incremental value of fused hybrid imaging \\
\hline Gaemperli et al. (2007) [50] & $\begin{array}{l}\text { SPECT/64-slice CTCA } \\
\text { and 3-D image fusion }\end{array}$ & $\begin{array}{l}38 \text { patients with } \geq 1 \\
\text { SPECT defect }\end{array}$ & $\begin{array}{l}\text { Modification of initial interpretation in } 29 \% \text { of } \\
\text { patients. In equivocal lesions, haemodynamic } \\
\text { relevance could be confirmed in } 35 \% \text { and } \\
\text { excluded in } 25 \%\end{array}$ \\
\hline Santana et al.(2009) [51] & $\begin{array}{l}\text { 16- and 64-slice CTCA and } \\
\text { MPI (SPECT or }{ }^{82} \text { Rb PET) }\end{array}$ & $\begin{array}{l}50 \text { patients with } \\
\text { suspected CAD }\end{array}$ & $\begin{array}{l}\text { Modification of initial interpretation in } 28 \% \text { of } \\
\text { patients. Trend towards increased sensitivity } \\
\text { (by } 17 \% \text { ) in patients with MVD }\end{array}$ \\
\hline Slomka et al. (2009) [45] & $\begin{array}{l}\text { Motion-frozen SPECT/64-slice } \\
\text { CTCA (automatic coregistration) }\end{array}$ & $\begin{array}{l}35 \text { patients with } \\
\text { suspected CAD }\end{array}$ & $\begin{array}{l}\text { Improved diagnostic performance in RCA and } \\
\text { LCX territories }\end{array}$ \\
\hline
\end{tabular}

SPECT single photon emission computed tomography, CTCA CT coronary angiography, MPI myocardial perfusion imaging, PET positron emission tomography, $C A D$ coronary artery disease, $R C A$ right carotid artery, $L C X$ left circumflex artery

prognostic information is provided by the spatial superposition of perfusion defects and coronary CT stenosis [53]. In this study, 324 consecutive patients undergoing hybrid SPECT/ CTCA were subdivided into three groups: (1) stenosis by CTCA and matching reversible SPECT defect (i.e. the perfusions defect was superimposed on the stenotic coronary artery on the hybrid 3-D display), (2) unmatched CTCA and SPECT finding and (3) normal finding by CTCA and SPECT. On follow-up (median 2.8 years), a corresponding matched hybrid finding was associated with a significantly higher event rate [death or myocardial infarction (MI)] and proved to be an independent predictor for major adverse cardiovascular events (MACE). The annual death/MI rate was 6.0,2.8 and 1.3 \% for patients with matched, unmatched and normal findings.

Two further studies have proven that hybrid imaging has an impact on downstream resource utilization and most notably on revascularization rates. Pazhenkottil et al. reported that revascularization rates within 60 days were 41, 11 and $0 \%$ for matched, unmatched and normal findings, respectively $(p<0.001)$ [54]. In a similar study by Schaap and colleagues, revascularization rates were even higher with 90, 31 and $0 \%$ for matched, unmatched and normal findings, respectively $(p<0.001)$ [55]. These results should be highlighted in the context of the recent SPARC study which documented a surprisingly modest impact of stand-alone non-invasive imaging modalities on downstream resource utilization with 38$61 \%$ of patients with the most severe abnormalities on CTCA or perfusion scans not being referred for invasive angiography [56]. Thus, it appears that the combined anatomical and functional assessment of CAD by hybrid imaging may encourage guideline-concordant patient management among physicians ordering those tests.

\section{Clinical indications for hybrid imaging}

Open questions remain as to which patients benefit from integrated hybrid imaging. It appears that the vast majority of patients referred for non-invasive cardiac imaging studies to exclude or confirm the presence of CAD are adequately addressed with one single study (i.e. either CTCA or functional imaging test). Additionally, the increased radiation and higher costs of hybrid imaging studies have to be considered as an important factor. Therefore, patients should be carefully selected where an added value of hybrid imaging may be anticipated.

\section{Intermediate pretest likelihood}

The pretest likelihood of CAD can be assessed based on the age and gender of the subject, the typicality of chest pain and the ECG changes on exercise testing [57]. Patients with intermediate pretest likelihood are more likely to yield intermediate or inconclusive results in one of the imaging studies, e.g. intermediate coronary stenosis on CTCA. In this situation, an additional perfusion study may address the haemodynamic relevance of such lesions and help to decide whether the patient can be treated medically or needs to be referred for invasive angiography with revascularization. Such sequential hybrid imaging protocols have been implemented in several centres with hybrid imaging capabilities. However, beta blockers given prior to the CTCA study may affect the subsequent SPECT study by blunting perfusion defect extent and reversibility, and vasodilator stress protocols should be preferred over exercise or dobutamine whenever the CTCA study precedes the SPECT $[58,59]$. Likewise, CT tomographs having a high temporal resolution obviating the need for beta blocker administration prior to the imaging study are preferred.

\section{Multivessel disease}

Patients with MVD represent a population that is at increased risk for future cardiac events and therefore is often referred for revascularization to improve outcomes. However, the majority of patients with angiographic three-vessel disease turn out 
to have considerably less flow-limiting lesions than suspected from their angiograms [7]. Compared to perfusion imaging alone, hybrid imaging may have an incremental diagnostic value to identify haemodynamically significant lesions particularly in patients with MVD (Fig. 3) [51]. By this means, hybrid imaging may allow targeted revascularization procedures in MVD patients avoiding stent or bypass overuse with the inherent adverse sequelae of stent or graft reocclusion.

\section{Significant side branch disease}

Side branches are often regarded as prognostically insignificant but may be associated with significant angina. Although perfusion defects in side branch disease are generally smaller, they may be difficult to distinguish from main branch disease. Hybrid imaging provides accurate coregistration of coronary anatomy and perfusion defects and therefore allows identifcation of flow-limiting lesions in side branches (diagonal, posterolateral branches) for targeted revascularization procedures [50] (Fig. 4).

\section{Chronic total occlusions}

Percutaneous revascularization procedures in patients with chronic total occlusions (CTO) are associated with higher rates of complications and larger contrast media and radiation administration. Therefore, proof of myocardial ischaemia in the territory of the occluded vessel is mandatory prior to embarking on any potentially harmful intervention. On the one hand, hybrid imaging may accurately detect a perfusion defect located in the territory of the CTO vessel. On the other hand, anatomical CT information such as the morphology of the stump, the presence of side vessels, the length of occlusion and/or calcifications or the presence of tortuosity are predictors
Fig. 3 Example of a 59-year-old gentleman with three-vessel disease who underwent stenting of the right coronary artery a few months prior to his hybrid SPECT/CT study. Angiography revealed a long $80 \%$ stenosis of the mid left anterior descending artery and a $70 \%$ stenosis of the proximal left circumflex artery (LCX) which was confirmed on CTCA (yellow arrows, $\mathbf{c}$ ). SPECT showed a partially reversible perfusion defect (white arrows) of the inferolateral wall (a stress, b rest). On hybrid SPECT/CT, the inferolateral ischaemia could be localized to the LCX (e, arrows), while the anterior wall did not show any relevant perfusion defect (d). This indicates that treatment should be targeted to the LCX to reduce ischaemic burden of the LV myocardium

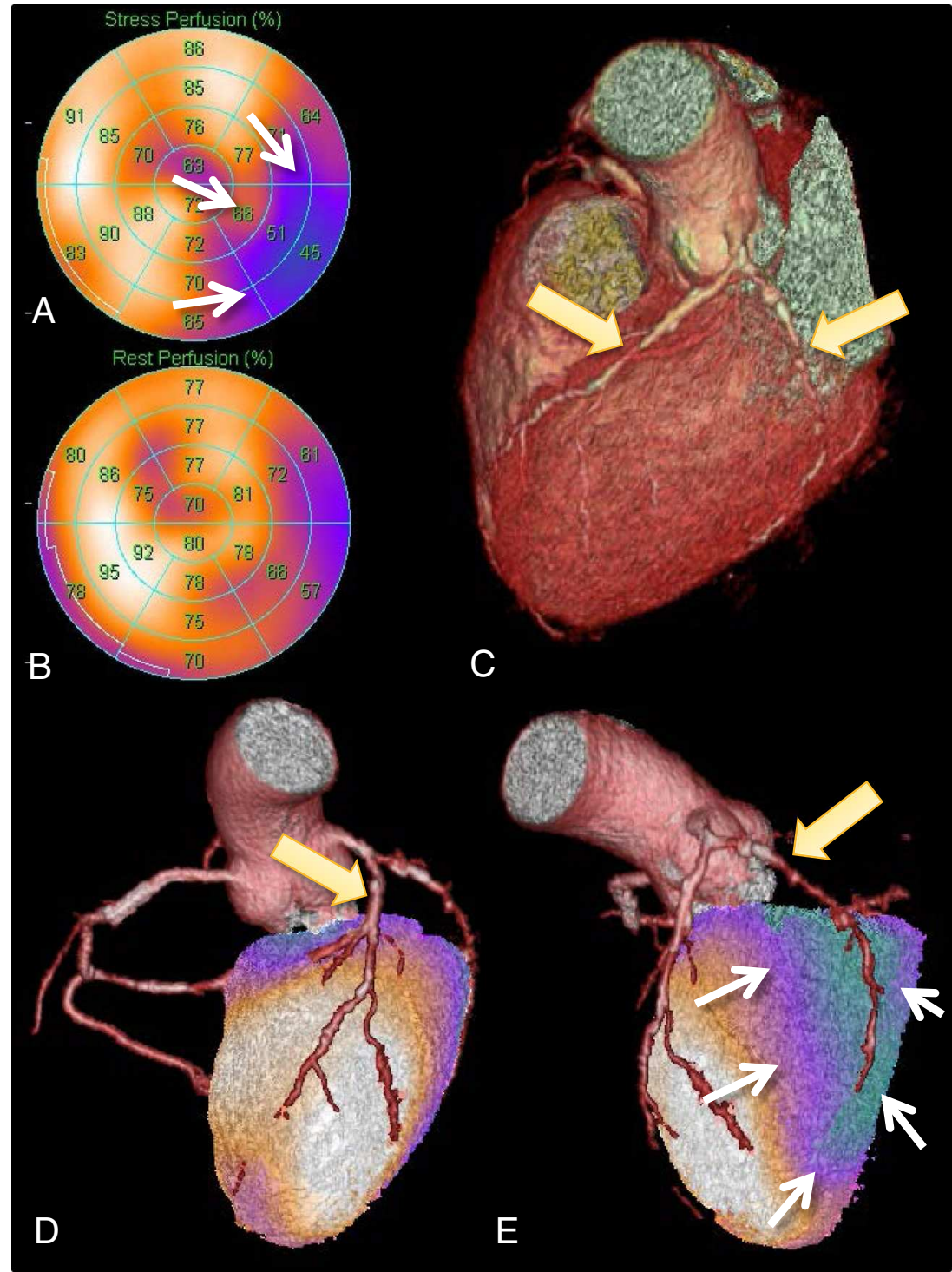


Fig. 4 A 67-year-old patient complained of new-onset chest pain after percutaneous coronary intervention (PCI) of his left anterior descending artery (LAD). a SPECT revealed a small reversible perfusion defect in the anterior basal myocardium (arrows). b Hybrid SPECT/CT shows stents in the LAD. The anterior ischaemia (arrows) is not subtended by the LAD, but by an overstented and occluded diagonal branch that is not visible on CT angiography (dotted yellow line). Review of coronary angiography before (c) and after (d) stenting of the $\mathrm{LAD}$ reveals inadvertent closure of the diagonal branch after PCI (arrows)

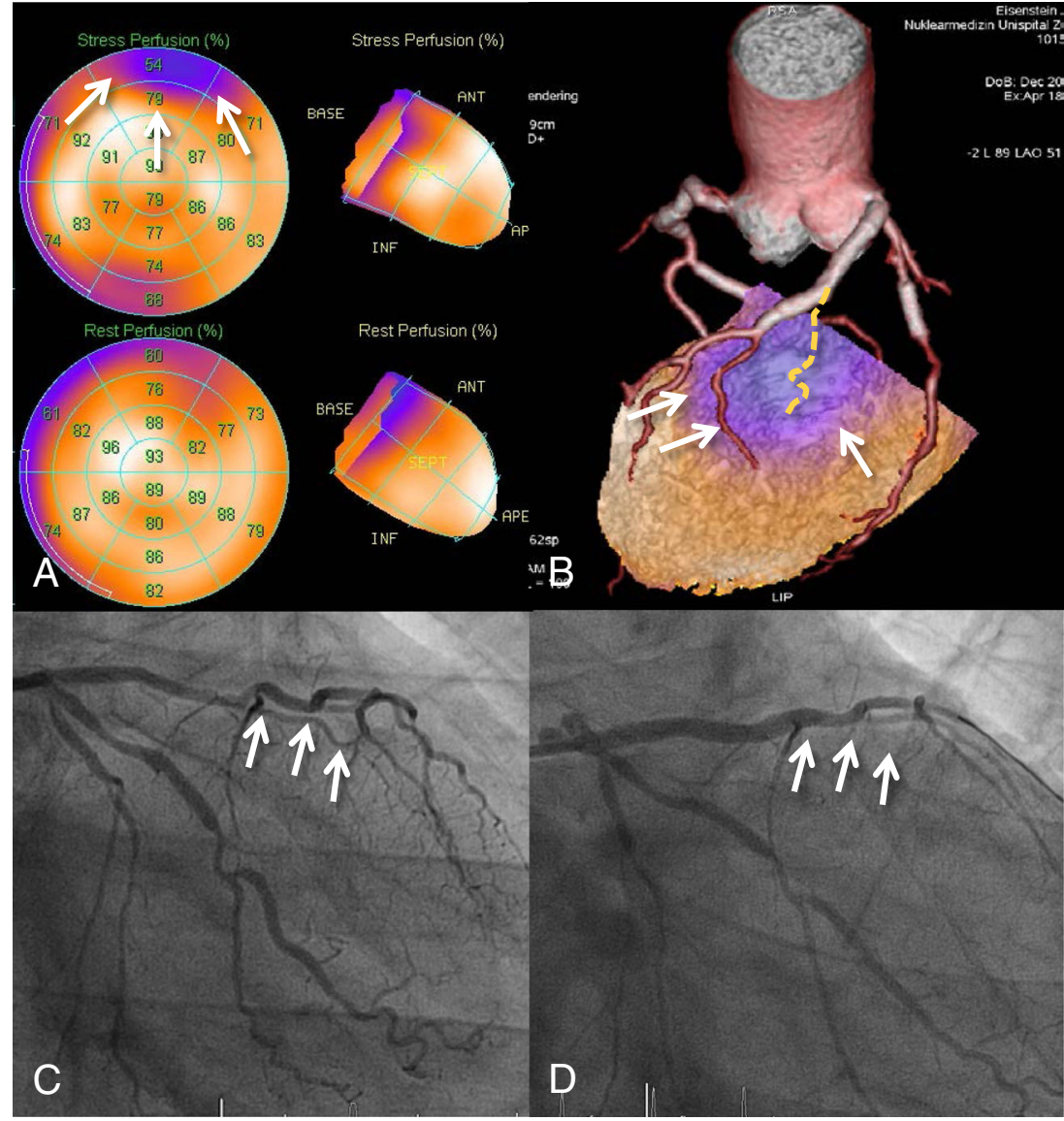

of procedural success and may help to decide between anterograde or retrograde revascularization approaches [60].

\section{Hybrid imaging for attenuation correction}

The quality and accuracy of nuclear perfusion studies is often affected by non-uniform photon attenuation, a common source of image artefacts with often unpredictable patterns. Whereas the accuracy of cardiac PET imaging has long benefited from correction methods for tissue attenuation, in SPECT imaging, commercial methods have only recently been made available. Various techniques with different line sources such as ${ }^{241} \mathrm{Am},{ }^{153} \mathrm{Gd}$ or ${ }^{99 \mathrm{~m}} \mathrm{Tc}$ have been proposed but yielded ambiguous results [61].

CT has shown promising results for attenuation correction of SPECT allowing consistent image reading [62, 63]. The radiation emitted from X-ray $\mathrm{CT}$ tubes is polychromatic compared to the monoenergetic gamma photons from a radioactive line source. Also, the flux of photons from a typical X-ray tube is much higher than a conventional sealed radioactive source. As a result transmission images for attenuation correction can be obtained within seconds using CT. However, some pitfalls of CT-based attenuation correction should be considered. For example, misalignment between SPECT and the attenuation map can lead to artefacts in the apical, septal and anterior walls that will appear as defects. It also can cause overcorrection in the basal inferior and lateral segments. There is evidence that mismatches along the other directions may have a similar effect.

Although in SPECT photon energies are lower $(70 \mathrm{keV}$ with ${ }^{210} \mathrm{Tl}$ and $140 \mathrm{keV}$ with ${ }^{99 \mathrm{~m}} \mathrm{Tc}$ versus $511 \mathrm{keV}$ with positron-emitting radionuclides) and thus more susceptible to attenuation, the pattern of attenuation is less predictable than with PET. Therefore, with SPECT, the use of X-raybased attenuation correction is less well established. As a result, most current dedicated cardiac SPECT systems are not equipped with a low-dose CT facility for attenuation correction. This can be overcome by using low-dose native coronary artery calcium score (CACS) scans performed on a stand-alone high-end CT device for attenuation correction of the SPECT images. In contrast to present low-dose CT facilities of hybrid SPECT/CT systems, CACS scans are ECGtriggered and acquired during a single breath-hold. Hence, this approach requires the use of interface software for manual coregistration of SPECT and CT information to avoid misalignment between both images (Fig. 5). The feasibility and reproducibility of this method has been demonstrated by Schepis and co-workers using CACS scans during full inspiratory and expiratory breath-hold [64]. Parametric attenuation 
Fig. 5 Coregistration of lowdose CT (a) and SPECT (b) for attenuation correction of myocardial perfusion SPECT. The fused SPECT/CT display (c) allows one to identify misalignment and correct it by realigning the SPECT with regard to the CT images. (Reprinted with permission from Springer [64])

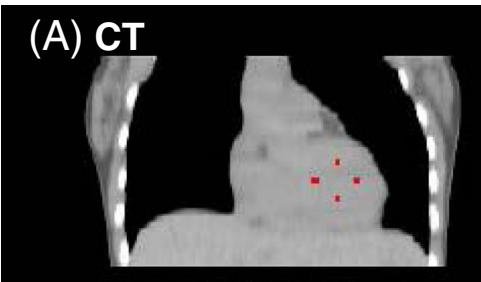

CT Coronals

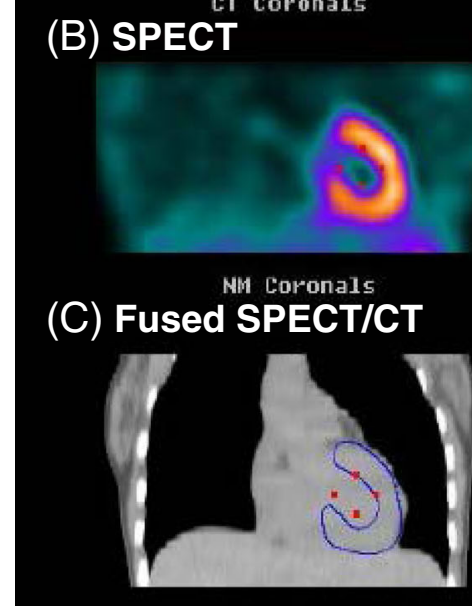

NM Loronals

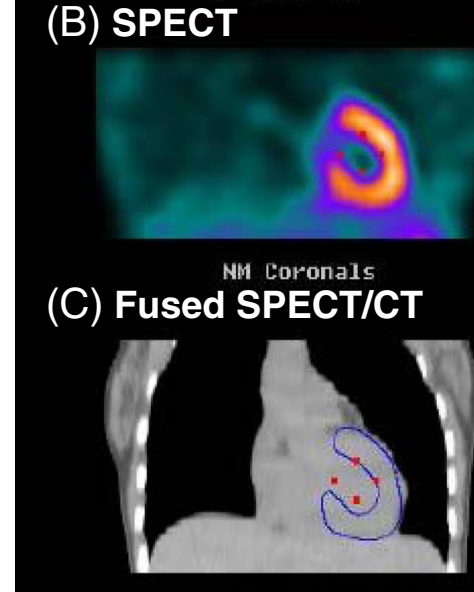

Fused Eorona15

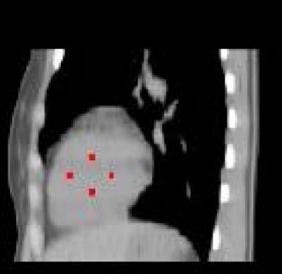

c1 sagitta15

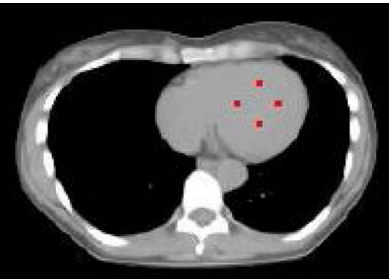

ct Transaxials

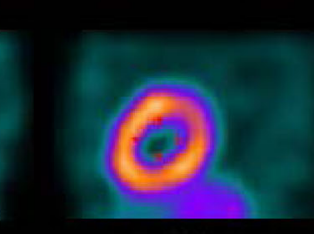

NM Sagittals

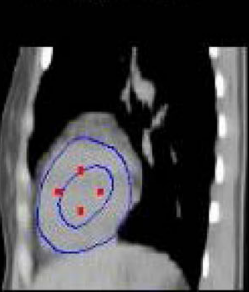

Fused Sagittals

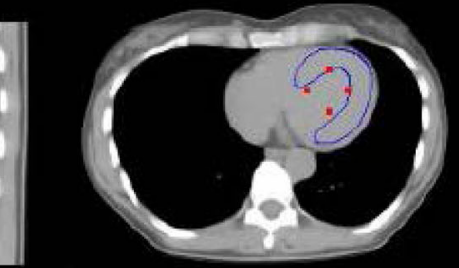

Fused Transaxials maps from CACS scans provided accurate and reliable attenuation correction of SPECT images resulting in a very good correlation compared to the established attenuation correction with the low-dose low-resolution CT facility included in the hybrid SPECT/CT system.

\section{Added value of CACS over SPECT alone}

CACS provides an estimate of coronary atherosclerotic plaque burden and correlates strongly with the overall amount of coronary plaque (calcified and non-calcified) seen in postmortem examinations [65]. Assessment of the presence of subclinical coronary atherosclerosis with CACS provides an opportunity to identify asymptomatic patients who are at risk for developing clinical CAD over the long term. Several studies in asymptomatic subjects have consistently shown that CACS provides accurate risk estimates for cardiac death and ischaemic events beyond clinical parameters $[66,67]$. These observations have led to the implementation of CACS in current recommendations for coronary risk assessment [68, 69]. Particularly in patients at intermediate clinical risk, CACS may prove helpful in more accurate risk stratification [70]. In addition to its prognostic value, CACS may also offer diagnostic information. Coronary artery calcifications are almost always present with angiographically significant CAD; thus, a CACS of 0 virtually excludes any significant angiographic CAD [71], particularly in asymptomatic patients. However, specificity and
PPV (23-62 \%) are low and increase only moderately when shifting the CACS cut-off from 0 to higher values.

While CACS is rarely used as a diagnostic test for CAD detection, combined protocols with SPECT may be attractive through integration of anatomical and functional information. In a recent study by Schepis and colleagues the combination of SPECT and CACS resulted in a significant improvement in sensitivity and specificity for the diagnosis of angiographically significant CAD compared to SPECT alone [9]. The presence of high atherosclerotic burden in a symptomatic patient with a normal or near-normal perfusion scan should alert the reader to the possibility of MVD and a potential underestimation of jeopardized myocardium by SPECT alone [72]. On the other hand, an equivocal perfusion result in the presence of zero CACS may raise the suspicion of an attenuation or respiratory artefact justifying further $\mathrm{CTCA}$ to rule out $\mathrm{CAD}$.

CACS may also add prognostic information to MPI. Schenker and colleagues observed an independent stepwise increase in death and MI rates with increasing CACS in 695 consecutive patients regardless of the presence or absence of ischaemia on perfusion scan [73]. Among patients with normal perfusion, the annualized event rate in patients with a CACS of 0 was $2.6 \%$ versus $12.3 \%$ in those with a CACS $\geq 1,000$. Likewise, in patients with ischaemia, the annualized event rate in those with a CACS of 0 was lower than among patients with a CACS $\geq 1,000$ (8.2 versus $22.1 \%$ ). Similar results were obtained in 510 asymptomatic patients, albeit at a lower CACS cut-off of 100 [74]. These results were not 
confirmed in a similar study performed in 1,153 very low-risk patients undergoing SPECT and CACS with short follow-up [75]. Chang and colleagues reported an incremental prognostic value of CACS in 977 asymptomatic patients with normal SPECT perfusion scans and a follow-up of 6.9 years [76]. There was a 3.6-fold relative increase for any cardiac event and a 2.8-fold relative increase for death/MI when the CACS was severe $(>400)$ versus minimal $(\leq 10)$. Separation of the survival curves occurred at 3 years after initial testing for all cardiac events and at 5 years for death/MI. This indicates that if CACS is severe the "warranty period" of a normal SPECT expires after 3-5 years. The REPROSPECT registry revealed the added prognostic value of fusing CACS and myocardial perfusion SPECT to allocate calcified vessels to their respective vascular territories [77]. Patients were divided into three groups (1) MPI defect matched by calcification $(C A C \geq 1)$ in the subtending coronary artery, (2) unmatched MPI and CT finding and (3) normal finding by MPI and CT. At the 35month follow-up, the most unfavourable outcome was observed in patients with matched findings (36 versus $16 \%$ in the group with unmatched findings versus $0 \%$ in the normal group, $p<0.001$ ) (Fig. 6).

\section{Perspectives of hybrid imaging}

Hybrid imaging is increasingly contributing to the development of imaging strategies which go beyond the assessment of myocardial perfusion. Furthermore, the use of other modalities (e.g. cardiac MRI) may expand existing indications for hybrid imaging. A number of novel hybrid imaging

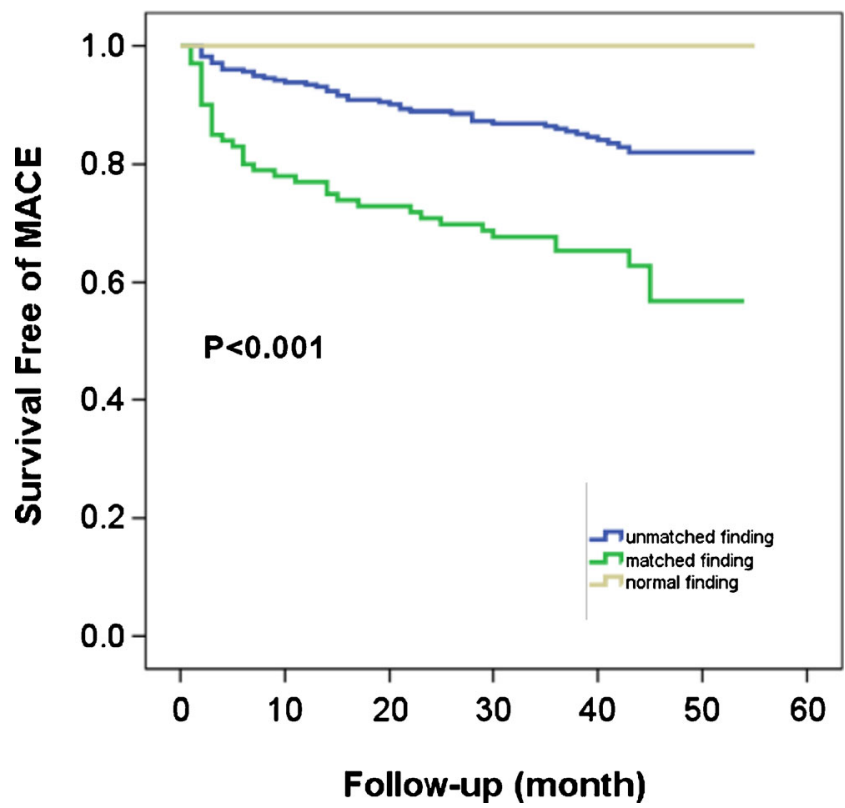

Fig. 6 Kaplan-Meier survival curves free of MACE according to normal, unmatched and matched findings of SPECT and CACS on CT. (Reprinted with permission from Oxford University Press [77]) approaches hold promise to make their way into clinical practice or are already being used in very specialized centres.

Cardiac resynchronization therapy (CRT) has established itself as an important therapeutic option to improve symptoms and prognosis in heart failure patients. Unfortunately, CRT non-responder rates are still high (approximately $30 \%$ ), particularly in patients with ischaemic heart disease. Therefore, efficient tools to predict CRT success and improve patient selection are urgently needed. Hybrid imaging may combine venous anatomy from $\mathrm{CT}$ (by scanning in the venous phase) with viability information from SPECT. This approach may allow visualization of venous morphology and identification of candidate veins for percutaneous lead insertion, while at the same time viability in this territory is ascertained [78]. It is likely that patients in whom the LV lead is placed in a nonviable territory may not respond to CRT. Furthermore, mechanical LV dyssynchrony can be quantified by phase analysis of gated perfusion SPECT studies [79]. Potentially, this approach could prove useful to optimize CRT.

Experimental approaches in human and animal studies have used hybrid imaging to colocalize anatomical information with (mainly radionuclide) signals from molecular pathways that precede or underlie changes in morphology or pathophysiology. Examples are the use of neuronal imaging to identify subjects at risk for ventricular arrhythmia, the development of compounds targeting plaque vulnerability before rupture and subsequent MI, the targeting of biomechanisms which precede LV remodelling and heart failure development, the development of reporter gene imaging techniques and the implementation of cell labelling for imaging of engraftment after transplantation [80, 81].

\section{Conclusion}

A comprehensive appraisal of anatomical and functional aspects of disease is crucial in cardiac imaging, particularly for the appropriate management of patients with known or suspected CAD. Hybrid imaging has entered the clinical arena in the past few decades and is facing increasing interest for clinical and research purposes as it allows one to obtain combined anatomical and functional information of cardiac pathology through non-invasive multimodality imaging. Hybrid SPECT/CT imaging is promoted by the dissemination of hybrid devices, the development of improved devices and protocols with reduced radiation exposure and shorter acquisition times, and the implementation of efficient and dedicated image reconstruction and fusion algorithms. The combined anatomical and functional information on CAD extent and severity may facilitate diagnosis, improve risk stratification and guide appropriate management strategies (particularly by selecting patients that may benefit from revascularization procedures) in particular subsets of CAD patients. However, the added costs and increased radiation exposure of hybrid 
imaging studies must be considered before ordering such tests, and larger prospective studies are needed to address issues such as incremental clinical value, impact on patient management and outcome and cost-effectiveness. Nevertheless, the use of hybrid imaging in other fields of cardiology may enhance our pathophysiological understanding of cardiac pathologies by linking morphological changes with underlying biochemical or subsequent functional alterations and thereby open avenues for hybrid imaging in heart failure, CRT, stem cell and gene therapy, plaque imaging, cardiac remodelling and regenerative medicine.

\section{Conflicts of interest None.}

\section{References}

1. Fazel R, Krumholz HM, Wang Y, Ross JS, Chen J, Ting HH, et al. Exposure to low-dose ionizing radiation from medical imaging procedures. N Engl J Med 2009;361:849-57.

2. Murthy VL, Naya M, Foster CR, Hainer J, Gaber M, Di Carli G, et al. Improved cardiac risk assessment with noninvasive measures of coronary flow reserve. Circulation 2011;124:2215-24.

3. Gaemperli O, Kaufmann PA. Why quantify myocardial perfusion? Curr Cardiovasc Imaging Rep 2012;5:133-43.

4. Gould KL, Johnson NP. Coronary artery disease: percent stenosis in CAD-a flaw in current practice. Nat Rev Cardiol 2010;7:482-4.

5. Uren NG, Melin JA, De Bruyne B, Wijns W, Baudhuin T, Camici PG. Relation between myocardial blood flow and the severity of coronary-artery stenosis. N Engl J Med 1994;330:1782-8.

6. White CW, Wright CB, Doty DB, Hiratza LF, Eastham CL, Harrison $\mathrm{DG}$, et al. Does visual interpretation of the coronary arteriogram predict the physiologic importance of a coronary stenosis? N Engl J Med 1984;310:819-24.

7. Tonino PA, Fearon WF, De Bruyne B, Oldroyd KG, Leesar MA, Ver Lee $\mathrm{PN}$, et al. Angiographic versus functional severity of coronary artery stenoses in the FAME study fractional flow reserve versus angiography in multivessel evaluation. J Am Coll Cardiol 2010;55: 2816-21.

8. Hacker M, Jakobs T, Hack N, Nikolaou K, Becker C, von Ziegler F, et al. Sixty-four slice spiral CT angiography does not predict the functional relevance of coronary artery stenoses in patients with stable angina. Eur J Nucl Med Mol Imaging 2007;34:4-10.

9. Schepis T, Gaemperli O, Koepfli P, Namdar M, Valenta I, Scheffel H, et al. Added value of coronary artery calcium score as an adjunct to gated SPECT for the evaluation of coronary artery disease in an intermediate-risk population. J Nucl Med 2007;48:1424-30.

10. Gaemperli O, Schepis T, Valenta I, Koepfli P, Husmann L, Scheffel $\mathrm{H}$, et al. Functionally relevant coronary artery disease: comparison of 64-section CT angiography with myocardial perfusion SPECT. Radiology 2008;248:414-23.

11. Schuijf JD, Wijns W, Jukema JW, Atsma DE, de Roos A, Lamb HJ, et al. Relationship between noninvasive coronary angiography with multi-slice computed tomography and myocardial perfusion imaging. J Am Coll Cardiol 2006;48:2508-14.

12. Boden WE, O'Rourke RA, Teo KK, Hartigan PM, Maron DJ, Kostuk WJ, et al. Optimal medical therapy with or without PCI for stable coronary disease. N Engl J Med 2007;356:1503-16.

13. BARI 2D Study Group, Frye RL, August P, Brooks MM, Hardison $\mathrm{RM}$, Kelsey SF, et al. A randomized trial of therapies for type 2 diabetes and coronary artery disease. N Engl J Med 2009;360: 2503-15.

14. Hachamovitch R, Hayes SW, Friedman JD, Cohen I, Berman DS. Comparison of the short-term survival benefit associated with revascularization compared with medical therapy in patients with no prior coronary artery disease undergoing stress myocardial perfusion single photon emission computed tomography. Circulation 2003;107: 2900-7.

15. Shaw LJ, Berman DS, Maron DJ, Mancini GB, Hayes SW, Hartigan PM, et al. Optimal medical therapy with or without percutaneous coronary intervention to reduce ischemic burden: results from the Clinical Outcomes Utilizing Revascularization and Aggressive Drug Evaluation (COURAGE) trial nuclear substudy. Circulation 2008;117:1283-91.

16. Tonino PA, De Bruyne B, Pijls NH, Siebert U, Ikeno F, van't Veer M, et al. Fractional flow reserve versus angiography for guiding percutaneous coronary intervention. N Engl J Med 2009;360:213-24.

17. De Bruyne B, Pijls NH, Kalesan B, Barbato E, Tonino PA, Piroth Z, et al. Fractional flow reserve-guided PCI versus medical therapy in stable coronary disease. N Engl J Med 2012;367:991-1001.

18. Fox K, Garcia MA, Ardissino D, Buszman P, Camici PG, Crea F, et al. Guidelines on the management of stable angina pectoris: executive summary: the Task Force on the Management of Stable Angina Pectoris of the European Society of Cardiology. Eur Heart J 2006;27: 1341-81.

19. Task Force on Myocardial Revascularization of the European Society of Cardiology (ESC) and the European Association for CardioThoracic Surgery (EACTS), European Association for Percutaneous Cardiovascular Interventions (EAPCI), Wijns W, Kolh P, Danchin N, Di Mario C, et al. Guidelines on myocardial revascularization. Eur Heart J 2010;31:2501-55.

20. Shaw LJ, Hachamovitch R, Berman DS, Marwick TH, Lauer MS, Heller GV, et al. The economic consequences of available diagnostic and prognostic strategies for the evaluation of stable angina patients: an observational assessment of the value of precatheterization ischemia. Economics of Noninvasive Diagnosis (END) Multicenter Study Group. J Am Coll Cardiol 1999;33:661-9.

21. Min JK, Leipsic J, Pencina MJ, Berman DS, Koo BK, van Mieghem $\mathrm{C}$, et al. Diagnostic accuracy of fractional flow reserve from anatomic CT angiography. JAMA 2012;308:1237-45.

22. Budoff MJ, Dowe D, Jollis JG, Gitter M, Halamert E, Scherer M, et al. Diagnostic performance of 64-multidetector row coronary computed tomographic angiography for evaluation of coronary artery stenosis in individuals without known coronary artery disease: results from the prospective multicenter ACCURACY (Assessment by Coronary Computed Tomographic Angiography of Individuals Undergoing Invasive Coronary Angiography) trial. J Am Coll Cardiol 2008;52:1724-32.

23. Sharma A, Arbab-Zadeh A. Assessment of coronary heart disease by CT angiography: current and evolving applications. J Nucl Cardiol 2012;19:796-806.

24. Vanhoenacker PK, Heijenbrok-Kal MH, Van Heste R, Decramer I, Van Hoe LR, Wijns W, et al. Diagnostic performance of multidetector CT angiography for assessment of coronary artery disease: metaanalysis. Radiology 2007;244:419-28.

25. von Ballmoos MW, Haring B, Juillerat P, Alkadhi H. Metaanalysis: diagnostic performance of low-radiation-dose coronary computed tomography angiography. Ann Intern Med 2011;154: 413-20.

26. Alkadhi H, Stolzmann P, Desbiolles L, Baumueller S, Goetti R, Plass A, et al. Low-dose, 128-slice, dual-source CT coronary angiography: accuracy and radiation dose of the high-pitch and the step-and-shoot mode. Heart 2010;96:933-8.

27. Moscariello A, Takx RA, Schoepf UJ, Renker M, Zwerner PL, O'Brien TX, et al. Coronary CT angiography: image quality, diagnostic accuracy, and potential for radiation dose reduction using a 
novel iterative image reconstruction technique-comparison with traditional filtered back projection. Eur Radiol 2011;21:2130-8.

28. Morsbach F, Desbiolles L, Plass A, Leschka S, Schmidt B, Falk V, et al. Stenosis quantification in coronary $\mathrm{CT}$ angiography: impact of an integrated circuit detector with iterative reconstruction. Invest Radiol 2013;48:32-40.

29. Schuhbaeck A, Achenbach S, Layritz C, Eisentopf J, Hecker F, Pflederer $\mathrm{T}$, et al. Image quality of ultra-low radiation exposure coronary CT angiography with an effective dose $<0.1 \mathrm{mSv}$ using high-pitch spiral acquisition and raw data-based iterative reconstruction. Eur Radiol 2013;23:597-606.

30. Maurovich-Horvat P, Hoffmann U, Vorpahl M, Nakano M, Virmani $\mathrm{R}$, Alkadhi $\mathrm{H}$. The napkin-ring sign: CT signature of high-risk coronary plaques? JACC Cardiovasc Imaging 2010;3:440-4.

31. Seifarth H, Schlett CL, Nakano M, Otsuka F, Károlyi M, Liew G, et al. Histopathological correlates of the napkin-ring sign plaque in coronary CT angiography. Atherosclerosis 2012;224:90-6.

32. Maurovich-Horvat P, Schlett CL, Alkadhi H, Nakano M, Otsuka F, Stolzmann $\mathrm{P}$, et al. The napkin-ring sign indicates advanced atherosclerotic lesions in coronary CT angiography. JACC Cardiovasc Imaging 2012;5:1243-52.

33. Otsuka K, Fukuda S, Tanaka A, Nakanishi K, Taguchi H, Yoshikawa $\mathrm{J}$, et al. Napkin-ring sign on coronary $\mathrm{CT}$ angiography for the prediction of acute coronary syndrome. JACC Cardiovasc Imaging 2013;6:448-57.

34. Klocke FJ, Baird MG, Lorell BH, Bateman TM, Messer JV, Berman DS, et al. ACC/AHA/ASNC guidelines for the clinical use of cardiac radionuclide imaging-executive summary: a report of the American College of Cardiology/American Heart Association Task Force on Practice Guidelines (ACC/AHA/ASNC Committee to Revise the 1995 Guidelines for the Clinical Use of Cardiac Radionuclide Imaging). J Am Coll Cardiol 2003;42:1318-33.

35. Iskander $\mathrm{S}$, Iskandrian AE. Risk assessment using single-photon emission computed tomographic technetium- $99 \mathrm{~m}$ sestamibi imaging. J Am Coll Cardiol 1998;32:57-62.

36. Hachamovitch R, Rozanski A, Shaw LJ, Stone GW, Thomson LE, Friedman JD, et al. Impact of ischaemia and scar on the therapeutic benefit derived from myocardial revascularization vs. medical therapy among patients undergoing stress-rest myocardial perfusion scintigraphy. Eur Heart J 2011;32:1012-24.

37. Slomka PJ, Dey D, Duvall WL, Henzlova MJ, Berman DS, Germano G. Advances in nuclear cardiac instrumentation with a view towards reduced radiation exposure. Curr Cardiol Rep 2012;14:208-16.

38. Husmann L, Herzog BA, Gaemperli O, Tatsugami F, Burkhard N, Valenta I, et al. Diagnostic accuracy of computed tomography coronary angiography and evaluation of stress-only single-photon emission computed tomography/computed tomography hybrid imaging: comparison of prospective electrocardiogram-triggering vs. retrospective gating. Eur Heart J 2009;30:600-7.

39. Manka R, Kuhn FP, Kuest SM, Gaemperli O, Kozerke S, Kaufmann PA. Hybrid cardiac magnetic resonance/computed tomographic imaging: first fusion of three-dimensional magnetic resonance perfusion and low-dose coronary computed tomographic angiography. Eur Heart J 2011;32:2625.

40. Donati OF, Alkadhi H, Scheffel H, Kuehnel C, Hennemuth A, Wyss $\mathrm{C}$, et al. 3D fusion of functional cardiac magnetic resonance imaging and computed tomography coronary angiography: accuracy and added clinical value. Invest Radiol 2011;46:331-40.

41. Stolzmann P, Alkadhi H, Scheffel H, Hennemuth A, Kuehnel C, Baumueller S, et al. Image fusion of coronary CT angiography and cardiac perfusion MRI: a pilot study. Eur Radiol 2010;20: 1174-9.

42. Herzog BA, Buechel RR, Katz R, Brueckner M, Husmann L, Burger IA, et al. Nuclear myocardial perfusion imaging with a cadmiumzinc-telluride detector technique: optimized protocol for scan time reduction. J Nucl Med 2010;51:46-51.
43. Valenta I, Treyer V, Husmann L, Gaemperli O, Schindler MJ, Herzog BA, et al. New reconstruction algorithm allows shortened acquisition time for myocardial perfusion SPECT. Eur J Nucl Med Mol Imaging 2010;37:750-7.

44. Gaemperli O, Schepis T, Kalff V, Namdar M, Valenta I, Stefani L, et al. Validation of a new cardiac image fusion software for threedimensional integration of myocardial perfusion SPECT and standalone 64-slice CT angiography. Eur J Nucl Med Mol Imaging 2007;34:1097-106.

45. Slomka PJ, Cheng VY, Dey D, Woo J, Ramesh A, Van Kriekinge S, et al. Quantitative analysis of myocardial perfusion SPECT anatomically guided by coregistered 64-slice coronary CT angiography. J Nucl Med 2009;50:1621-30.

46. Namdar M, Hany TF, Koepfli P, Siegrist PT, Burger C, Wyss CA, et al. Integrated PET/CT for the assessment of coronary artery disease: a feasibility study. J Nucl Med 2005;46:930-5.

47. Rispler S, Keidar Z, Ghersin E, Roquin A, Soil A, Dragu R, et al. Integrated single-photon emission computed tomography and computed tomography coronary angiography for the assessment of hemodynamically significant coronary artery lesions. J Am Coll Cardiol 2007;49:1059-67.

48. Sato A, Nozato T, Hikita H, Miyazaki S, Takahashi Y, Kuwahara T, et al. Incremental value of combining 64-slice computed tomography angiography with stress nuclear myocardial perfusion imaging to improve noninvasive detection of coronary artery disease. J Nucl Cardiol 2010;17:19-26.

49. Kajander S, Joutsiniemi E, Saraste M, Pietilä M, Ukkonen H, Saraste A, et al. Cardiac positron emission tomography/computed tomography imaging accurately detects anatomically and functionally significant coronary artery disease. Circulation 2010;122: 603-13.

50. Gaemperli O, Schepis T, Valenta I, Husmann L, Scheffel H, Duerst V, et al. Cardiac image fusion from stand-alone SPECT and CT: clinical experience. J Nucl Med 2007;48:696-703.

51. Santana CA, Garcia EV, Faber TL, Sirineni GK, Esteves FP, Sanyal $\mathrm{R}$, et al. Diagnostic performance of fusion of myocardial perfusion imaging (MPI) and computed tomography coronary angiography. J Nucl Cardiol 2009;16:201-11.

52. van Werkhoven JM, Schuijf JD, Gaemperli O, Jukema JW, Boersma E, Wijns W, et al. Prognostic value of multislice computed tomography and gated single-photon emission computed tomography in patients with suspected coronary artery disease. J Am Coll Cardiol 2009;53:623-32.

53. Pazhenkottil AP, Nkoulou RN, Ghadri JR, Herzog BA, Buechel RR, Küest SM, et al. Prognostic value of cardiac hybrid imaging integrating single-photon emission computed tomography with coronary computed tomography angiography. Eur Heart J 2011;32: 1465-71.

54. Pazhenkottil AP, Nkoulou RN, Ghadri JR, Herzog BA, Küest SM, Husmann L, et al. Impact of cardiac hybrid single-photon emission computed tomography/computed tomography imaging on choice of treatment strategy in coronary artery disease. Eur Heart J 2011;32: 2824-9.

55. Schaap J, de Groot JA, Nieman K, Meijboom WB, Boekholdt SM, Post MC, et al. Hybrid myocardial perfusion SPECT/CT coronary angiography and invasive coronary angiography in patients with stable angina pectoris lead to similar treatment decisions. Heart 2013;99:188-94.

56. Hachamovitch R, Nutter B, Hlatky MA, Shaw LJ, Ridner ML, Dorbala $\mathrm{S}$, et al. Patient management after noninvasive cardiac imaging results from SPARC (Study of myocardial perfusion and coronary anatomy imaging roles in coronary artery disease). $\mathrm{J}$ Am Coll Cardiol 2012;59:462-74.

57. Diamond GA, Forrester JS. Analysis of probability as an aid in the clinical diagnosis of coronary-artery disease. N Engl J Med 1979;300:1350-8. 
58. Müller-Suur R, Eriksson SV, Strandberg LE, Mesko L. Comparison of adenosine and exercise stress test for quantitative perfusion imaging in patients on beta-blocker therapy. Cardiology 2001;95:112-8.

59. Reyes E, Stirrup J, Roughton M, D’Souza S, Underwood SR, Anagnostopoulos C. Attenuation of adenosine-induced myocardial perfusion heterogeneity by atenolol and other cardioselective betaadrenoceptor blockers: a crossover myocardial perfusion imaging study. J Nucl Med 2010;51:1036-43.

60. Stahli BE, Bonassin F, Goetti R, Küest SM, Frank M, Altwegg LA, et al. Coronary computed tomography angiography indicates complexity of percutaneous coronary interventions. J Invasive Cardiol 2012;24:196-201.

61. Corbett JR, Ficaro EP. Attenuation corrected cardiac perfusion SPECT. Curr Opin Cardiol 2000;15:330-6.

62. Utsunomiya D, Tomiguchi S, Shiraishi S, Yamada K, Honda T, Kawanaka K, et al. Initial experience with X-ray CT based attenuation correction in myocardial perfusion SPECT imaging using a combined SPECT/CT system. Ann Nucl Med 2005;19:485-9.

63. Fricke E, Fricke H, Weise R, Kammeier A, Hagedorn R, Lotz N, et al. Attenuation correction of myocardial SPECT perfusion images with low-dose CT: evaluation of the method by comparison with perfusion PET. J Nucl Med 2005;46:736-44.

64. Schepis T, Gaemperli O, Koepfli P, Rüegg C, Burger C, Leschka S, et al. Use of coronary calcium score scans from stand-alone multislice computed tomography for attenuation correction of myocardial perfusion SPECT. Eur J Nucl Med Mol Imaging 2007;34:11-9.

65. Rumberger JA, Simons DB, Fitzpatrick LA, Sheedy PF, Schwartz RS. Coronary artery calcium area by electron-beam computed tomography and coronary atherosclerotic plaque area. A histopathologic correlative study. Circulation 1995;92:2157-62.

66. Budoff MJ, Shaw LJ, Liu ST, Weinstein SR, Mosler TP, Tseng PH, et al. Long-term prognosis associated with coronary calcification: observations from a registry of 25,253 patients. J Am Coll Cardiol 2007:49:1860-70

67. Detrano R, Guerci AD, Carr JJ, Bild DE, Burke G, Folsom AR, et al. Coronary calcium as a predictor of coronary events in four racial or ethnic groups. N Engl J Med 2008;358:1336-45.

68. Berman DS, Hachamovitch R, Shaw LJ, Friedman JD, Hayes SW, Thomson LE, et al. Roles of nuclear cardiology, cardiac computed tomography, and cardiac magnetic resonance: noninvasive risk stratification and a conceptual framework for the selection of noninvasive imaging tests in patients with known or suspected coronary artery disease. J Nucl Med 2006;47:1107-18.

69. Grundy SM, Cleeman JI, Merz CN, Brewer HB, Clark LT, Hunninghake DB, et al. Implications of recent clinical trials for the National Cholesterol Education Program Adult Treatment Panel III guidelines. Circulation 2004;110:227-39.

70. Greenland P, Bonow RO, Brundage BH, Budoff MJ, Eisenberg MJ, Grundy SM, et al. ACCF/AHA 2007 clinical expert consensus document on coronary artery calcium scoring by computed tomography in global cardiovascular risk assessment and in evaluation of patients with chest pain: a report of the American College of Cardiology Foundation Clinical Expert Consensus Task Force (ACCF/AHA
Writing Committee to Update the 2000 Expert Consensus Document on Electron Beam Computed Tomography) developed in collaboration with the Society of Atherosclerosis Imaging and Prevention and the Society of Cardiovascular Computed Tomography. J Am Coll Cardiol 2007;49:378-402.

71. Haberl R, Becker A, Leber A, Knez A, Becker C, Lang C, et al. Correlation of coronary calcification and angiographically documented stenoses in patients with suspected coronary artery disease: results of 1,764 patients. J Am Coll Cardiol 2001;37:451-7.

72. Ghadri JR, Pazhenkottil AP, Nkoulou RN, Goetti R, Buechel RR, Husmann L, et al. Very high coronary calcium score unmasks obstructive coronary artery disease in patients with normal SPECT MPI. Heart 2011;97:998-1003.

73. Schenker MP, Dorbala S, Hong EC, Rybicki FJ, Hachamovitch R, Kwong RY, et al. Interrelation of coronary calcification, myocardial ischemia, and outcomes in patients with intermediate likelihood of coronary artery disease: a combined positron emission tomography/ computed tomography study. Circulation 2008;117:1693-700.

74. Anand DV, Lim E, Hopkins D, Corder R, Shaw LJ, Sharp P, et al. Risk stratification in uncomplicated type 2 diabetes: prospective evaluation of the combined use of coronary artery calcium imaging and selective myocardial perfusion scintigraphy. Eur Heart J 2006;27:713-21.

75. Rozanski A, Gransar H, Wong ND, Shaw LJ, Miranda-Peats R, Polk $\mathrm{D}$, et al. Clinical outcomes after both coronary calcium scanning and exercise myocardial perfusion scintigraphy. J Am Coll Cardiol 2007;49:1352-61.

76. Chang SM, Nabi F, Xu J, Peterson LE, Achari A, Pratt CM, et al. The coronary artery calcium score and stress myocardial perfusion imaging provide independent and complementary prediction of cardiac risk. J Am Coll Cardiol 2009;54:1872-82.

77. Ghadri JR, Fiechter M, Fuchs TA, Scherrer A, Stehli J, Gebhard $\mathrm{C}$, et al. Registry for the Evaluation of the PROgnostic value of a novel integrated imaging approach combining Single Photon Emission Computed Tomography with coronary calcification imaging (REPROSPECT). Eur Heart J Cardiovasc Imaging 2013;14: 374-80.

78. van der Hoeven BL, Schalij MJ, Delgado V. Multimodality imaging in interventional cardiology. Nat Rev Cardiol 2012;9:333-46.

79. Pazhenkottil AP, Buechel RR, Husmann L, Nikoulou RN, Wolfrum M, Ghadri JR, et al. Long-term prognostic value of left ventricular dyssynchrony assessment by phase analysis from myocardial perfusion imaging. Heart 2011;97:33-7.

80. Kramer CM, Sinusas AJ, Sosnovik DE, French BA, Bengel FM. Multimodality imaging of myocardial injury and remodeling. J Nucl Med 2010;51 Suppl 1:107S-21S.

81. Camici PG, Rimoldi OE, Gaemperli O, Libby P. Non-invasive anatomic and functional imaging of vascular inflammation and unstable plaque. Eur Heart J 2012;33:1309-17.

82. Groves AM, Speechly-Dick ME, Kayani I, Pugliese F, Endozo R, McEwan J, et al. First experience of combined cardiac PET/64detector CT angiography with invasive angiographic validation. Eur J Nucl Med Mol Imaging 2009;36:2027-33. 